\title{
Preoperative Visualized Ultrasound Assessment of the Recurrent Laryngeal Nerve in Thyroid Cancer Surgery: Reliability and Risk Features by Imaging
}

\author{
Yushuang $\mathrm{He}^{1}$ \\ Zhihui $\mathrm{Li}^{2}$ \\ Yujia Yang' \\ Jianyong Lei ${ }^{2}$ \\ Yulan Peng' \\ 'Department of Ultrasound, West China \\ Hospital of Sichuan University, Chengdu, \\ Sichuan Province, 6I004I, People's \\ Republic of China; ${ }^{2}$ Department of \\ Thyroid Surgery, West China Hospital of \\ Sichuan University, Chengdu, Sichuan \\ Province, 6I004I, People's Republic of \\ China
}

\section{Correspondence: Yulan Peng}

Department of Ultrasound, West China Hospital of Sichuan University, No. 37

Guo Xue Xiang, Chengdu, Sichuan

Province, 61004I, People's Republic of

China

Tel +8618980601606

Fax +86288542 3192

Email yulanpeng520@। 26.com

Zhihui Li

Department of Thyroid Surgery, West

China Hospital of Sichuan University,

No. 37 Guo Xue Xiang, Chengdu, Sichuan

Province, 6I004I, People's Republic of

China

Tel +8618980602027

Fax +86 28 85422933

Email rockoliver@vip.sina.com
Background: Preoperative identification and visualization of tumor infiltration of the recurrent laryngeal nerve (RLN) in patients with thyroid cancer is important. The purpose of our study was to evaluate the reliability and feasibility of preoperative assessment by ultrasound and to identify ultrasound imaging features potentially associated with tumor infiltration of the RLN.

Methods: In this prospective study, patients undergoing thyroid cancer surgery at our institution between August 2020 and January 2021 were included, and preoperative ultrasound visualization of the RLN and thyroid lesions was performed. RLN infiltration was also confirmed surgically in all cases. Five patients with enlarged lymph nodes were selected to undergo injection of carbon nanoparticles to confirm the correctness of RLN identification by preoperative ultrasound. The repeatability of RLN assessment by ultrasound was evaluated by comparing the correlation between pre- and intraoperative, intra- and inter-group assessments. Parameters of normal RLNs according to age, sex, and body mass index were established. Finally, ultrasound imaging features of patients with RLN tumor infiltration were analyzed to identify potential risk predictors.

Results: According to the ultrasonic assessment, RLNs of 70 patients appeared normal, while 14 of those patients appeared to be infiltrated by tumors. During surgery, the 70 cases of normal RLNs were confirmed, but only 8 of the 14 suspected cases of infiltration were confirmed. In all five patients injected with carbon nanoparticles, the location of RLNs adjacent to the marked lymph nodes observed by surgeons corresponded to the RLN location identified by preoperative ultrasound. The repeatability of RLN estimation varied from moderate to excellent. There were no significant differences in cross-sectional area, width, or thickness of normal RLNs according to age, sex, or body mass index. Indistinct margin with tumor, incontinuous shape as ultrasound features by the analysis of patients with surgically confirmed RLN infiltration were associated with tumor invasion.

Conclusion: We show that preoperative ultrasound can be applied to visualize the RLN and may help predict tumor infiltration of the RLN.

Keywords: recurrent laryngeal nerve, ultrasound, visualization, reliability

\section{Introduction}

The ability to detect thyroid neoplasms has increased greatly in the last few years, which translates to a higher number of treated patients. ${ }^{1-3}$ Traditional thyroidectomy, innovative thyroidectomy techniques (including robotic and transoral approaches), and thyroid radiofrequency ablation are the most common treatment options for thyroid neoplasms. ${ }^{4,5}$ Complication like recurrent laryngeal nerve 
(RLN) injury is of great concern for all types of therapeutic management. It may cause hoarseness, dysphagia, and even dyspnea, which may substantially affect quality of life. Therefore, preoperative identification and visualization of the RLN are potentially crucial for surgeons to evaluate RLN infiltration as well as assess voice and laryngeal function.

Laryngoscopy is currently the only available technique for preoperative RLN assessment, and it measures RLN functional status by evaluating vocal cord mobility. ${ }^{6}$ Despite the popularity of this method in the preoperative setting, it is invasive, it does not allow visualization, and it lacks the sensitivity to directly detect tumor infiltration of the RLN. Therefore, alternative non-invasive techniques for RLN visualization are needed in thyroid surgery.

High-frequency ultrasound is widely used to localize and visualize peripheral nerves for diagnosis. ${ }^{7-9}$ However, ultrasound visualization of the RLN has rarely been reported, ${ }^{10}$ and the reliability of the measurements has not been evaluated. Therefore, whether ultrasound is feasible for preoperative RLN assessment in thyroid neoplasm surgery needs to be investigated.

An effective procedure for visualizing the RLN accurately and reliably by ultrasound still needs to be established. Generally, both RLNs pass along the tracheoesophageal groove. ${ }^{11}$ They penetrate the larynx at the level of the cricoid cartilage, which lies approximately at the level of the sixth cervical vertebra (C6). The C6 has a chassaignac tubercle, which can easily be identified in ultrasound. ${ }^{12,13}$ Therefore, the present study focused on the $\mathrm{C} 6-\mathrm{C} 7$ region.

The purpose of this study was to assess the feasibility and reliability of ultrasound for preoperative RLN visualization. We also aimed to determine normal ultrasound values for RLN width, thickness, and cross-sectional area. Finally, we evaluated thyroid lesions and surgically confirmed cases of RLN infiltration to identify ultrasound imaging features associated with such infiltration.

\section{Materials and Methods}

\section{Patient Selection}

This prospective study involved patients who had thyroid cancer confirmed by fine needle aspiration cytology and who were scheduled for thyroidectomy at our hospital between August 2020 and January 2021. All the study participants were Chinese. Patients underwent preoperative ultrasound examination to evaluate thyroid lesions and the RLNs. Patients were excluded if they had a history of thyroid or neck surgery, or if the RLN could not be traced by ultrasound.

\section{Preoperative Ultrasound Examination}

Ultrasound assessment was performed at the anterior cervical region (C6-C7 level) using a Philips EPIQ 7 device with a 5-12 MHz linear array transducer (Figure 1A-C). The examination was performed as follows. First, the patient lays supine, with the neck at maximal extension. The ultrasound probe was placed at the paratracheal area, parallel to the coronal body plane. The operator started to scan the thyroid to detect and evaluate lesion size, margin, location and relationship to the surrounding tissues. Then the RLNs were identified with the transducer in a transverse position, and color Doppler mode was used to distinguish the RLNs from small blood vessels. The RLN appeared as an oval, hypoechoic structure surrounded by hyperechoic tissue. From this position, the nerve was tracked upwards to the entry of the laryngeal area and downwards to the supraclavicular area, with slight adjustments of the scanning plane. Three static B-mode images were saved at distinct locations where the nerve structure could be clearly identified on one side of the patients' necks. Finally, the ultrasound transducer was rotated into a transverse position with respect to the long axis in order to further identify thyroid lesions and the RLNs. The same steps were performed on the other side. All patients were asked to relax and breathe slowly during the examination. The characteristics of lesions were recorded, as were the width (transverse diameter), thickness (anteroposterior diameter), and cross-sectional areas of both right and left RLNs. All scans were saved on the ultrasound device for off-line analysis.

\section{Carbon Nanoparticle Injection}

The particular features of the RLN make it difficult to label directly, so we opted to label the surrounding tissues. During the preoperative ultrasound scanning, five patients in our sample were found to have adenopathy in the central neck adjacent to the RLN. Since carbon nanoparticles are widely used in operations to trace lymph nodes, ${ }^{14}$ we selected one lymph node adjacent to the RLN in each of these patients for injection of carbon nanoparticles (Lai Mei Pharmaceutical Co, Chongqing, China). Injection was made in pre-operative room before the operations and the interval time from injection to surgery was less than 24 hours. The injection was 


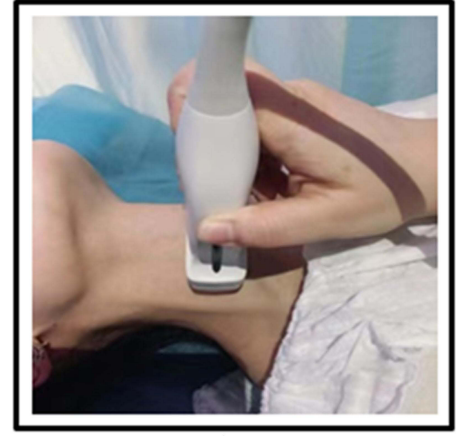

A

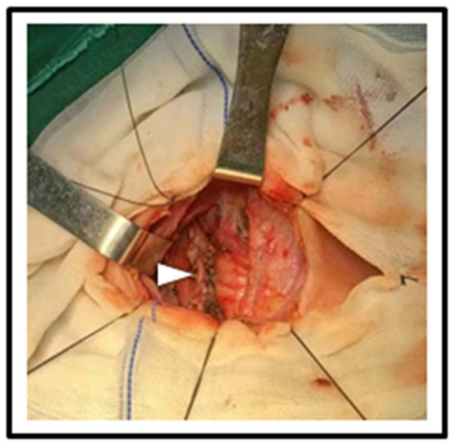

D

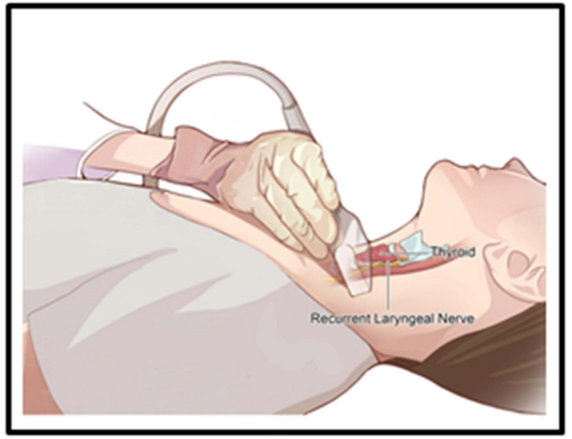

B

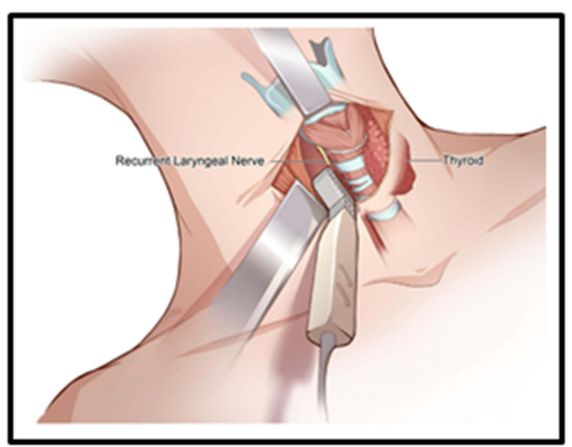

E
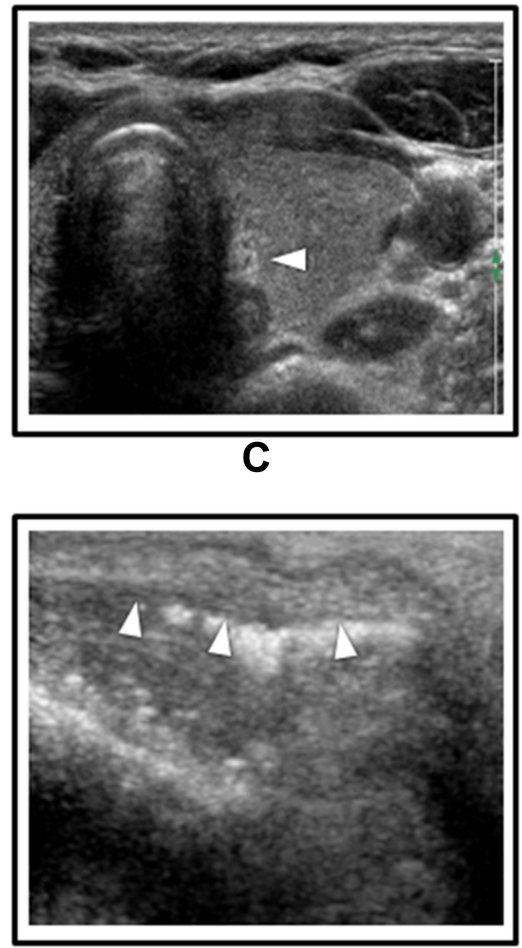

$\mathbf{F}$

Figure I Ultrasound visualization of the recurrent laryngeal nerve (RLN), preoperative (A-C) and intraoperative (D-F).

performed using a standard sterile technique under realtime ultrasound guidance with a Resona 7 ultrasonic system (Mindray Medical Solutions, Shenzhen, China) equipped with a 5-14 MHz linear array transducer. The operator had more than five years of experience in thyroid interventions. Before surgery, the operator used color Doppler ultrasound to plan the appropriate route to locate the assigned lymph nodes and to avoid injuring the vessels. Then, $0.1 \mathrm{~mL}$ of carbon nanoparticles were injected into the target lymph node using a free-hand, in-plane guidance technique. After injection, all patients were required to perform manual self-compression for 20 minutes.

\section{Invasion Confirmation and Intraoperative Ultrasound}

After the preoperative ultrasound assessment, all the enrolled patients underwent thyroid surgery, during which RLN invasion was assessed. Thyroidectomy was performed by experienced head and neck surgeons using standard procedures. When surgeons exposed the target surgical area, the marked lymph nodes were checked in order to confirm that the adhering RLNs were those that we had identified by preoperative ultrasound. As the surgery went on, the surgeons evaluated the relationship between thyroid lesions and the RLN. Confirmation of RLN invasion was defined as positive intraoperative findings or based on pathology of intraoperative cryosections.

Twenty patients without tumor invasion were selected at random to undergo intraoperative ultrasound measurements of RLN parameters as follows. Since the thyroid lobe with lesions had been completely removed, the RLN in the tracheoesophageal groove was exposed. Intraoperative ultrasound was performed using a Philips IU 22 system equipped with a $15-7 \mathrm{MHz}$ transducer. The probe was coated with acoustic gel, covered in a sterile sleeve, and placed directly on the RLN for measurement (Figure 1D-F). Because of the limitation of surgical incision, the thickness of the RLN was the only parameter measured longitudinally during the intraoperative ultrasound. Each RLN was measured three times, and the mean value was used in subsequent analyses.

The patients with confirmed infiltration of RLN were used to identify ultrasound imaging features of thyroid lesions and RLNs, which are potentially associated with tumor invasion. 


\section{Repeatability of RLN Visualization}

The repeatability of ultrasound visualization of the RLN was achieved by comparison of preoperative and intraoperative ultrasound, inter- and intra-group analyses, respectively. Inter- and intra-group analyses were assessed in two ways. In one approach, two ultrasonographers with more than five years of experience in performing ultrasound were trained in locating the RLN. They performed pre- and intraoperative measurements, independently of each other, on 20 patients without infiltration (see previous subsection). One ultrasonographer measured width, thickness, and cross-sectional area of RLN; then the second ultrasonographer, blinded to the work of the first, measured the same parameters. In a second approach, one ultrasonographer repeated these measurements after two months basing on the images stored in the ultrasound device for off-line analysis.

\section{Data Analysis}

Statistical analysis was performed with SPSS software (version 19.0; IBM, Armonk, NY, USA). RLN parameters were expressed as mean and standard deviation (SD), and differences were assessed for significance using an independent-samples paired $t$-test. Differences that were associated with $\mathrm{P}$ values lower than 0.05 were considered statistically significant.

Intra- and inter-observer repeatability was assessed using single-measure intraclass correlation coefficients (ICC) in a two-way random-effects model. ICC values $<0.40$ indicated poor agreement; values of $0.40-0.75$, moderate to good agreement; and values $>0.75$, excellent agreement. $^{15,16}$ Pearson's correlation test was used in the correlation analysis.

\section{Results}

\section{Clinical Characteristics of the Study Population}

Two hundred and eight patients were confirmed to have thyroid cancer by preoperative biopsy and enrolled for thyroidectomy at our hospital. According to the excluded criteria and the agreement of patients, only 84 patients were included in this study and received ultrasound evaluation of thyroid lesions and RLN. All the patients received thyroidectomy and all assessments of RLN infiltration or non-infiltration were confirmed by surgery. The majority of patients were females (56 females and 28 males) and the average age of the patients was $38.4 \pm 12.7$ years old. Tumor types were as follows: 80 cases were papillary carcinoma $(95.2 \%), 3$ were medullary carcinoma $(3.6 \%)$ and 1 was anaplastic carcinoma (1.2\%). RLN invasion was diagnosed by preoperative ultrasound in 14 patients, while the remaining 70 patients did not show ultrasound evidence of invasion. During the surgery, all 70 cases of RLNs without apparent invasion were confirmed to be normal, while 8 of the 14 cases of suspected tumor infiltration were confirmed.

\section{Confirmation of the RLN by Labeling Nearby Lymph Nodes}

In the five patients who were injected with carbon nanoparticles, RLNs exposed by surgeons were located near the labeled lymph nodes. The RLNs were confirmed to be the same RLNs that had been identified by preoperative ultrasound (Figure 2).

\section{Repeatability of RLN Visualization}

Pre- and intra-operative measurements are shown in Figure 3. The concordance of results between preoperative and intraoperative ultrasound parameters was moderate $(\mathrm{ICC}=0.403)$. The ICCs for measurements taken independently by two ultrasonographers ranged from 0.495 to 0.824 . The $\mathrm{R}^{2}$ value, also useful for assessing the correlation between two observers, ranged from 0.246 to 0.692 (Table 1). ICCs were also reasonably high for measurements of the same patients taken after two months; these ICCs ranged from 0.587 to 0.82 (Table 2).

\section{Average Parameter Values of Normal RLNs}

The mean cross-sectional area of the left RLN (4.35 $\pm 0.95 \mathrm{~mm})$ was similar to that of the right RLN (4.36 $\pm 0.95 \mathrm{~mm})$. The width of the two RLNs ranged from 2 to $2.7 \mathrm{~mm}$, and the thickness from 1.8 to $2.5 \mathrm{~mm}$. There were no significant differences in RLN cross-sectional area, width, or thickness by age, sex, or body mass index (Table 3).

\section{Ultrasound Imaging Features of Thyroid Cancer and RLNs Associated with Tumor Invasion}

During scanning, we assessed the risk of RLN tumor invasion based on ultrasound features. The RLN infiltration in this study was defined as follows: (i) the RLN adhered to a thyroid tumor, (ii) the boundary between the 

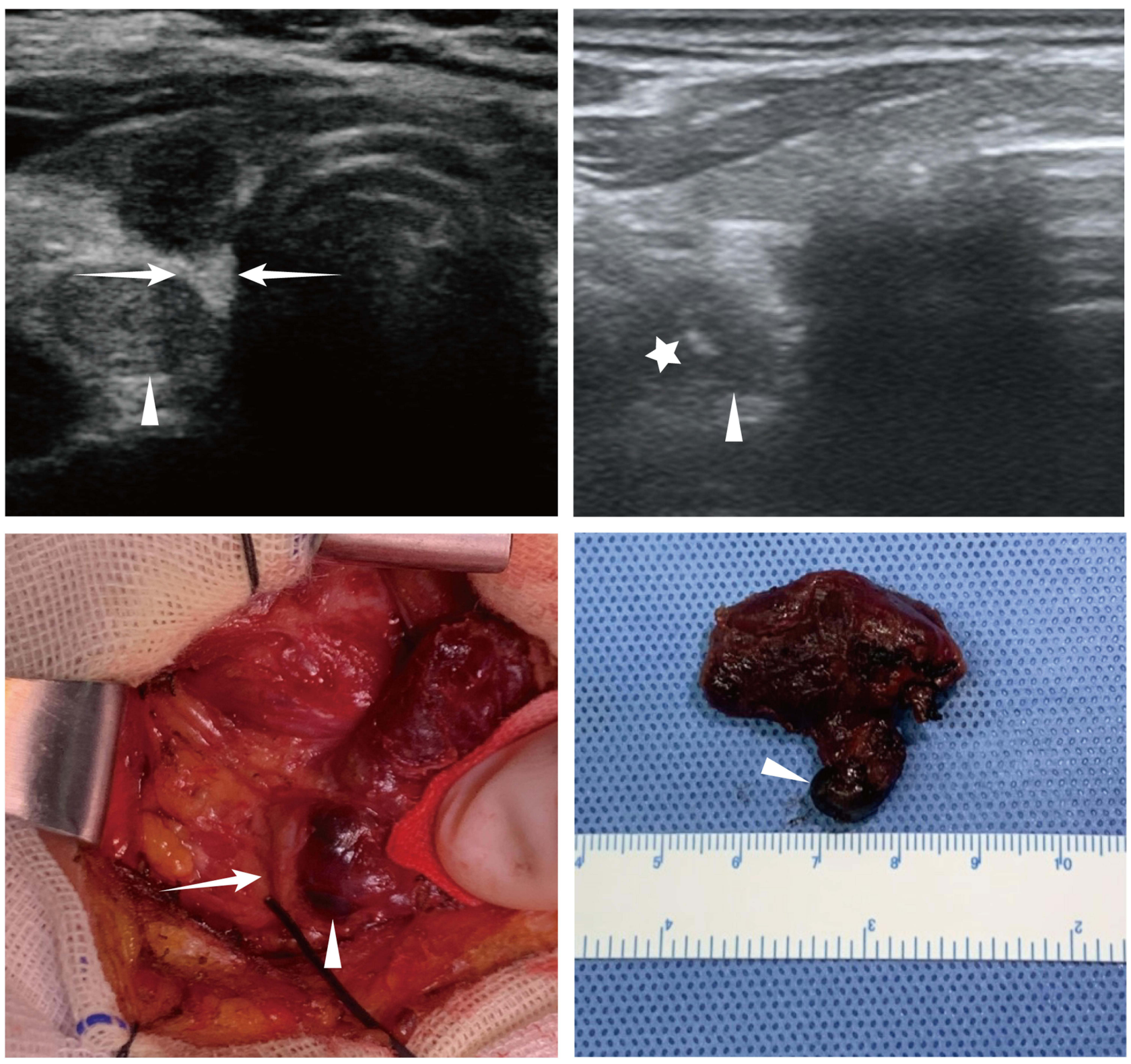

Figure 2 Confirmation of preoperative ultrasound assessment of the recurrent laryngeal nerve (RLN) based on intraoperative detection of adjacent lymph nodes marked with carbon nanoparticles (arrow, RLN; triangle, lymph node; pentagon, injection needle).

tumor and the nerve could not be clearly distinguished, and (iii) the longitudinal shape of the RLN was discontinuous. In our sample, 14 patients were suspected of RLN infiltration by preoperative ultrasound, and the suspicions were confirmed by surgical criteria in 8 patients. The largest tumor diameter ranged from 8 to $55 \mathrm{~mm}$, and the tumors in the 8 patients were located in the posterior focus of the thyroid, near the trachea, or as part of extrathyroidal extension. In the other six patients who were suspected of RLN infiltration based on ultrasound, tumors were located in the median and inferior focus of the thyroid. The RLN around Berry's ligament was more likely to be infiltrated among these patients whose thyroid lesions with largest diameter less than $2 \mathrm{~cm}$ (Table 4 and Figure 4).

\section{Discussion}

Ultrasound is a non-invasive, relatively inexpensive, and convenient visualization technique. It is widely used to assess peripheral nerves and can complement existing imaging algorithms. Our study, based on surgical verification of RLN invasion and nanoparticle labeling of nearby lymph nodes, suggests that preoperative ultrasound is a promising technique for visualizing the RLN before 


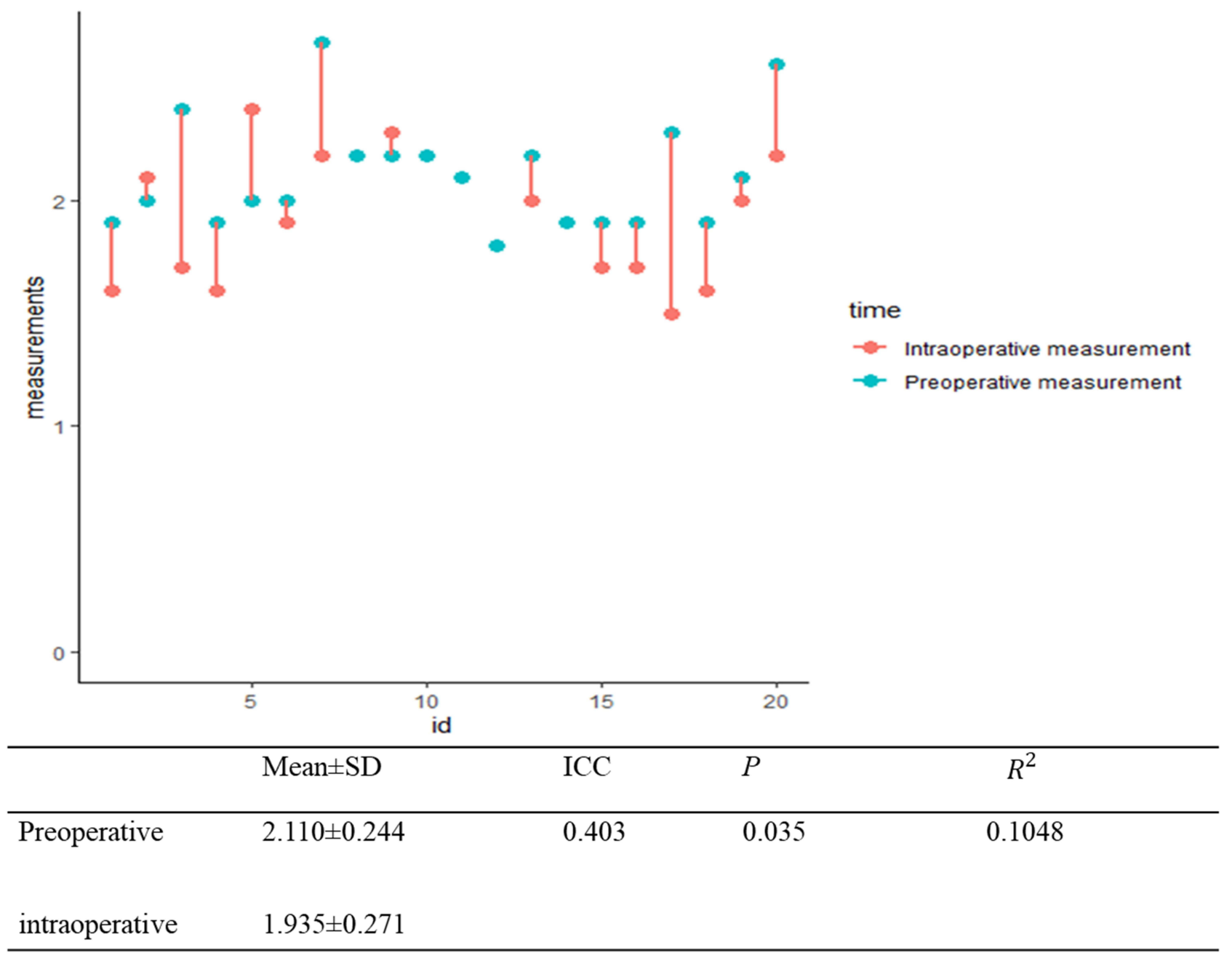

Figure 3 Comparison of pre- and intra-operative ultrasound measurement of recurrent laryngeal nerve (RLN).

thyroid surgery and for predicting tumor invasion of the nerve.

One of the main purposes of our study was to define a feasible procedure for correctly identifying and visualizing the RLN, and for distinguishing it from other nerves and structures in the neck. In particular, we wanted to ensure that we could follow the course of the nerve, and we found color Doppler mode to be effective for distinguishing the RLN from small blood vessels, since both structures can have similar morphology in gray-scale ultrasound. ${ }^{17}$ We confirmed the reliability of ultrasound localization by injecting, in a subset of patients, carbon nanoparticles into lymph nodes adjacent to the RLN. The marked lymph nodes, recognized during surgery, proved to be adjacent to the RLN identified by preoperative ultrasound, as expected.

We found no significant differences in RLN parameters as a function of demographic characteristics. These negative results should be interpreted with caution, given the small sample. We were aware of only one study reporting parameters of normal RLNs: ${ }^{18}$ that study reported the diameter of the right RLN to be $0.86 \pm 0.1 \mathrm{~mm}$ and the diameter of the left RLN to be $1.13 \pm 0.14 \mathrm{~mm}$, which are inconsistent with the diameters that we measured. Future studies should validate our findings.

We prospectively analyzed a small number of patients who were suspected of having RLN infiltration based on preoperative ultrasound. The accuracy of ultrasound diagnosis of tumor infiltration of the RLN was 57\% (8/14) based on the reference of positive findings intraoperatively or in pathology of intraoperative cryosections. We attribute the ultrasound-based misdiagnosis of invasion in six patients to insufficient experience of the operators and the small sample. Previously, only one study evaluated the possibility of predicting RLN invasion preoperatively using ultrasound. 
Table I Inter-Observer Reliability of the Measurements of Recurrent Laryngeal Nerve (RLN) Parameters

\begin{tabular}{|c|c|c|c|c|c|}
\hline \multirow[t]{2}{*}{ Variable } & \multicolumn{2}{|c|}{ Mean士SD } & \multirow[t]{2}{*}{ ICC } & \multirow[t]{2}{*}{$P$} & \multirow[t]{2}{*}{$R^{2}$} \\
\hline & Observer A & Observer B & & & \\
\hline CSA (R) & $3.760 \pm 0.972$ & $4.440 \pm 0.763$ & 0.648 & 0.001 & 0.4454 \\
\hline CSA (L) & $4.085 \pm 1.188$ & $4.675 \pm 1.027$ & 0.824 & $<0.001$ & 0.6925 \\
\hline Width (R) & $2.370 \pm 0.382$ & $2.510 \pm 0.325$ & 0.707 & $<0.001$ & 0.5124 \\
\hline Width (L) & $2.355 \pm 0.363$ & $2.460 \pm 0.422$ & 0.792 & $<0.001$ & 0.6407 \\
\hline Thickness (R) & $2.190 \pm 0.284$ & $2.210 \pm 0.269$ & 0.495 & 0.011 & 0.2460 \\
\hline Thickness (L) & $2.305 \pm 0.35 I$ & $2.315 \pm 0.345$ & 0.675 & $<0.001$ & 0.4563 \\
\hline
\end{tabular}

Notes: CSA values are expressed in $\mathrm{mm}^{2}$. The other values are expressed in $\mathrm{mm}$.

Abbreviations: SD, standard deviation; ICC, intraclass correlation coefficient; CSA, cross-sectional area; R, right RLN; L, left RLN.

Table 2 Intra-Observer Reliability of the Measurements of Recurrent Laryngeal Nerve (RLN) Parameters

\begin{tabular}{|c|c|c|c|c|c|}
\hline \multirow[t]{2}{*}{ Variable } & \multicolumn{2}{|c|}{ Mean \pm SD } & \multirow[t]{2}{*}{ ICC } & \multirow[t]{2}{*}{$P$} & \multirow[t]{2}{*}{$R^{2}$} \\
\hline & First Measurement & Second Measurement & & & \\
\hline CSA (R) & $4.100 \pm 0.903$ & $3.860 \pm 0.661$ & 0.603 & 0.002 & 0.4009 \\
\hline CSA (L) & $3.870 \pm 0.746$ & $3.920 \pm 0.654$ & 0.820 & $<0.001$ & 0.6845 \\
\hline Width (R) & $2.165 \pm 0.225$ & $2.260 \pm 0.308$ & 0.629 & 0.001 & 0.4356 \\
\hline Width (L) & $2.140 \pm 0.289$ & $2.135 \pm 0.223$ & 0.807 & $<0.001$ & 0.6957 \\
\hline Thickness (R) & $1.975 \pm 0.188$ & $2.010 \pm 0.210$ & 0.587 & 0.003 & 0.3488 \\
\hline Thickness (L) & $2.060 \pm 0.277$ & $2.045 \pm 0.218$ & 0.787 & $<0.001$ & 0.6562 \\
\hline
\end{tabular}

Notes: CSA values are expressed in $\mathrm{mm}^{2}$. The other values are expressed in $\mathrm{mm}$.

Abbreviations: SD, standard deviation; ICC, intraclass correlation coefficient; R, right RLN; L, left RLN; CSA, cross-sectional area.

Table 3 Normal Recurrent Laryngeal Nerve (RLN) Parameters, Stratified by Age, Sex, and Body Mass Index

\begin{tabular}{|c|c|c|c|c|c|c|c|c|c|c|}
\hline \multirow[t]{2}{*}{ Variable } & \multicolumn{2}{|c|}{ Age, $\mathbf{Y r}$} & \multirow[t]{2}{*}{$P$} & \multicolumn{2}{|c|}{ Sex } & \multirow[t]{2}{*}{$P$} & \multicolumn{2}{|c|}{ BMI, $\mathrm{kg} / \mathrm{m}^{2}$} & \multirow[t]{2}{*}{$P$} & \multirow{2}{*}{$\begin{array}{l}\text { Average } \\
\text { Value }\end{array}$} \\
\hline & $>50$ & $\leq \mathbf{5 0}$ & & Male & Female & & $\geq \mathbf{2 4}$ & $<24$ & & \\
\hline CSA (R) & $4.253 \pm 1.003$ & $4.389 \pm 0.949$ & 0.647 & $4.660 \pm 0.864$ & $4.219 \pm 0.970$ & 0.068 & $4.624 \pm 1.012$ & $4.220 \pm 0.898$ & 0.089 & $4.364 \pm 0.953$ \\
\hline CSA (L) & $4.300 \pm 1.206$ & $4.37 I \pm 0.860$ & 0.802 & $4.634 \pm 0.961$ & $4.225 \pm 0.819$ & 0.080 & $4.612 \pm 1.025$ & $4.217 \pm 0.843$ & 0.087 & $4.358 \pm 0.924$ \\
\hline Width (R) & $2.376 \pm 0.457$ & $2.434 \pm 0.386$ & 0.633 & $2.486 \pm 0.308$ & $2.393 \pm 0.433$ & 0.360 & $2.393 \pm 0.340$ & $2.440 \pm 0.428$ & 0.636 & $2.424 \pm 0.397$ \\
\hline Width (L) & $2.274 \pm 0.405$ & $2.323 \pm 0.296$ & 0.622 & $2.417 \pm 0.299$ & $2.263 \pm 0.314$ & 0.055 & $2.380 \pm 0.303$ & $2.276 \pm 0.320$ & 0.190 & $2.314 \pm 0.316$ \\
\hline Thickness (R) & $2.135 \pm 0.367$ & $2.196 \pm 0.334$ & 0.561 & $2.274 \pm 0.269$ & $2.14 I \pm 0.362$ & 0.124 & $2.274 \pm 0.366$ & $2.135 \pm 0.315$ & 0.099 & $2.185 \pm 0.338$ \\
\hline Thickness (L) & $2.204 \pm 0.45$ I & $2.243 \pm 0.281$ & 0.689 & $2.313 \pm 0.269$ & $2.198 \pm 0.332$ & 0.153 & $2.286 \pm 0.315$ & $2.208 \pm 0.316$ & 0.325 & $2.236 \pm 0.316$ \\
\hline
\end{tabular}

Notes: CSA values are expressed in $\mathrm{mm}^{2}$. The other values are expressed in $\mathrm{mm}$.

Abbreviations: BMI, body mass index; CSA, cross-sectional area; R, right RLN; L, left RLN.

We propose that the three ultrasound features of tumor location (in the posterior focus of the thyroid, near the trachea, or in the extrathyroidal extension), indistinct ultrasound boundary between RLNs and tumors, and discontinuous longitudinal shape of RLNs are linked to risk of tumor infiltration of the RLNs. If all the above image features are presented in one case, the risk of invasion of the RLN is high. The TNM stage of tumor may rise to T4 (infiltration of RLN) and that may influence the treatment strategies. For example, if the RLNs were infiltrated even with normal vocal cord mobility, the patients could not be suitable for minimally invasive thyroid surgery. They need a traditional thyroidectomy. Then, if preoperative ultrasound evaluation of RLNs presented high risk of infiltration, the surgeons would carefully assess the possibility of the preservation, section or reconstruction of the RLNs.

Interestingly, tumor invasion of the RLN around Berry's ligament was more likely among our patients with neoplasms smaller than $2 \mathrm{~cm}$. If tumor invasion occurred around the ligament of Berry, preserving the RLN can be difficult because only part of the nerve is visible. ${ }^{19}$ Preoperative ultrasound evaluation, in contrast, 
Table 4 Summary of Ultrasound Features of Thyroid Tumors and Recurrent Laryngeal Nerve (RLN) in Patients with Tumor Infiltration of the RLN

\begin{tabular}{|c|c|c|c|c|c|c|c|c|}
\hline $\begin{array}{ll}\text { Features } & \text { Patient No. }\end{array}$ & I & 2 & 3 & 4 & 5 & 6 & 7 & 8 \\
\hline \multicolumn{9}{|l|}{ Ultrasound features of the tumor } \\
\hline Largest diameter $(\mathrm{mm})$ & 26 & 9 & 19 & 15 & 55 & 14 & 11 & 8 \\
\hline Margin & Indistinct & Indistinct & Indistinct & Indistinct & Indistinct & Indistinct & Indistinct & Indistinct \\
\hline Taller than wider & Yes & Yes & No & Yes & No & Yes & Yes & Yes \\
\hline Longitudinal location & Median & Median & Median & Inferior & Whole gland & Inferior & Median & Median \\
\hline Sagittal location & Posterior & Posterior & Posterior & Posterior & Whole gland & Posterior & Posterior & Posterior \\
\hline Near cricoid cartilage & Yes & Yes & No & Yes & Yes & No & No & No \\
\hline Near trachea & Yes & Yes & Yes & Yes & Yes & Yes & Yes & Yes \\
\hline Near esophagus & Yes & No & No & No & Yes & No & No & Yes \\
\hline Extrathyroidal extension & Yes & Yes & Yes & Yes & Yes & Yes & Yes & Yes \\
\hline Multifocal or unifocal & Multifocal & Unifocal & Unifocal & Multifocal & Unifocal & Unifocal & Unifocal & Unifocal \\
\hline \multicolumn{9}{|l|}{ Ultrasound features of the RLN } \\
\hline Location & Left & Right & Right & Right & Left & Left & Left & Left \\
\hline Margin with tumor & Indistinct & Indistinct & Indistinct & Indistinct & Indistinct & Indistinct & Indistinct & Indistinct \\
\hline Continuous Shape & No & No & No & No & No & No & No & No \\
\hline Display the RLN around Berry's ligament & Not clear & Not clear & Not clear & Not clear & Not clear & Not clear & Not clear & Clear \\
\hline
\end{tabular}

may help surgeons detect RLN infiltration, especially invasion in the RLNs' entrance into the larynx. In such cases, the RLN should be carefully separated from the tumor in order to preserve nerve function. According to the available data, we evaluated the possibility of nerve invasion based on the imaging features (in the posterior focus of the thyroid, near the trachea, or in the extrathyroidal extension, indistinct ultrasound boundary between RLNs and tumors, and discontinuous longitudinal shape of RLNs), but the RLN infiltration in this aspect cannot be confirmed by ultrasound alone. The confirmation is based on the positive intraoperative findings. With further study of preoperative visualized ultrasound assessment of the recurrent laryngeal nerve, the diagnostic performance in the aspect may be improved.

We faced some difficulties during ultrasound scanning. During surgery, an incision of only 6-12 cm was made in a natural skin crease, and the incision length depended on characteristics of the patient and disease. ${ }^{19}$ As a result, the specially shaped intraoperative ultrasound probe could be placed in only one position parallel to the vertical body plane, and it could not be rotated. In addition, during the preoperative exam, the limited extension of the neck impeded transducer movements. Thus, in some patients, we needed to adjust the scan plane slightly with the transducer and sometimes even change probe frequency to improve image quality.
Our study presents other limitations. Our small, singlecenter sample may show selection bias, threatening its generalizability to other patient populations. The surgery lasted from 3 to 6 hours, which limited the number of intraoperative ultrasound cases we could collect. In addition, we were only able to measure one thickness parameter intraoperatively, because of the limited surgical incision and use of a specially shaped ultrasound probe. Finally, we were unable to "anchor" our ultrasound measurements relative to specific anatomical markers. All these factors likely contributed to the relatively low consistency between pre- and intraoperative measurements.

Despite these limitations, our study demonstrates the feasibility of ultrasound visualization of the RLN. Future work should focus on optimizing these procedures. By evaluating the imaging features of tumors and RLNs associated with infiltration, preoperative ultrasound, as a noninvasive and convenient technique, may make thyroid surgery much more effective.

\section{Conclusions}

Preoperative ultrasound for RLN assessment is feasible and shows promise for detecting tumor infiltration of the RLN. Especially when tumors are smaller than $2 \mathrm{~cm}$, the technique may facilitate detection of RLN infiltration around Berry's ligament. Physicians with intermediate ultrasound experience can reliably visualize most of the RLNs. We found no 

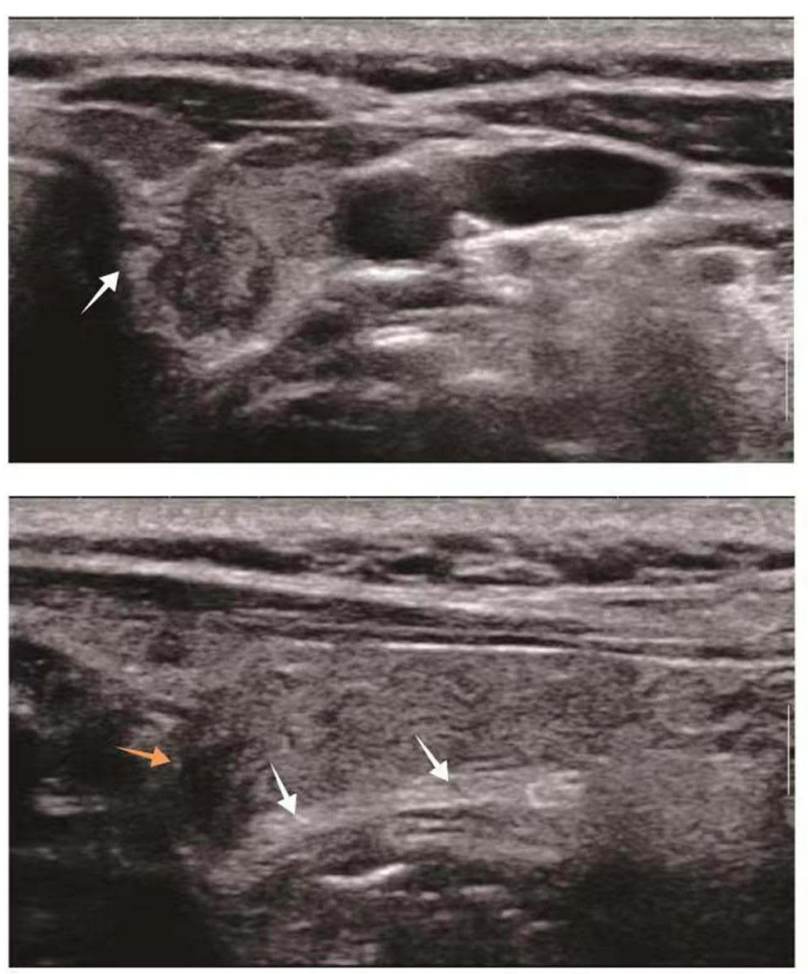

Figure 4 Gray-scale ultrasound image of a 65-year-old woman with a papillary thyroid tumor. The largest diameter of the tumor was $8 \mathrm{~mm}$, and its infiltration of the RLN around Berry's ligament was confirmed intraoperatively (white arrow, RLN; yellow arrow, thyroid tumor).

differences in ultrasound-based RLN parameters as a function of patient age, sex, or body mass index.

\section{Data Sharing Statement}

The data that support the findings of this study are available from the corresponding author upon reasonable request.

\section{Ethical Approval Statement}

The study was approved by the Medical Ethics Committee of West China Hospital, Sichuan University, and it was conducted in strict accordance with the principles of the Declaration of Helsinki (as revised in 2013). Before enrollment, patients were informed about the procedures, and written informed consent was obtained. A statement covering patient data confidentiality was fully respected during data collection and the preparation of the manuscript.

\section{Funding}

This research did not receive any specific grant from funding agencies in the public, commercially, or not-forprofit sectors.

\section{Disclosure}

The authors report no conflicts of interest related to this work.

\section{References}

1. Kitahara CM, Sosa JA. The changing incidence of thyroid cancer. Nat Rev Endocrinol. 2016;12(11):646-653. doi:10.1038/nrendo.2016.110

2. Cabanillas ME, McFadden DG, Durante C. Thyroid cancer. Lancet. 2016;388(10061):2783-2795. doi:10.1016/S0140-6736(16)30172-6

3. Sosa JA, Hanna JW, Robinson KA, et al. Increases in thyroid nodule fine-needle aspirations, operations, and diagnoses of thyroid cancer in the United States. Surgery. 2013;154:1420-1426. doi:10.1016/j. surg.2013.07.006

4. Wilhelm T, Metzig A. Endoscopic minimally invasive thyroidectomy (eMIT): a prospective proof-of-concept study in humans. World J Surg. 2011;35:543-551. doi:10.1007/s00268-010-0846-0

5. Tae K. Complications of transoral thyroidectomy: overview and update. Clin Exp Otorhinolaryngol. 2021;14:169-178. doi:10.21053/ ceo.2020.02110

6. Chandrasekhar SS, Randolph GW, Seidman MD. Clinical practice guideline: improving voice outcomes after thyroid surgery. Otolaryngol Head Neck Surg. 2013;148(6 suppl):S1-37. doi:10.1177/0194599813487301

7. Abraham A, Izenberg A, Dodig D, et al. Peripheral nerve ultrasound imaging shows enlargement of peripheral nerves outside the brachial plexus in neuralgic amyotrophy. J Clin Neurophysiol. 2016;33:e31e33. doi:10.1097/WNP.0000000000000304

8. $\mathrm{Ng}$ ES, Vijayan J, Therimadasamy A, et al. The added value of preoperative ultrasonography of the ulnar nerve: an observational study. Muscle Nerve. 2010;42:613-614. doi:10.1002/mus.21800

9. Samarawickrama D, Therimadasamy AK, Chan YC, et al. Nerve ultrasound in electrophysiologically verified tarsal tunnel syndrome. Muscle Nerve. 2016;53:906-912. doi:10.1002/mus.24963

10. Solbiati L, De Pra L, Lerace T, et al. High-resolution sonography of the recurrent laryngeal nerve: anatomic and pathologic considerations. AJR Am J Roentgenol. 1985;145:989-993. doi:10.2214/ajr.145.5.989

11. Myssiorek D. Recurrent laryngeal nerve paralysis: anatomy and etiology. Otolaryngol Clin North Am. 2004;37:25-44. doi:10.1016/ S0030-6665(03)00172-5

12. Gervasio A, Mujahed L, Biasio A, et al. Ultrasound anatomy of the neck: the infrahyoid region. $J$ Ultrasound. 2010;13:85-89. doi:10.1016/j.jus.2010.09.006

13. Chang KV, Wu WT, Özçakar L. Ultrasound-guided interventions of the cervical spine and nerves. Phys Med Rehabil Clin N Am. 2018;29:93-103. doi:10.1016/j.pmr.2017.08.008

14. Yu W, Xu G, Sun J, Zhong N. Carbon nanoparticles guide contralateral central neck dissection in patients with papillary thyroid cancer. Oncol Lett. 2018;16:447-452.

15. Arda K, Ciledag N, Aktas E, et al. Quantitative assessment of normal soft-tissue elasticity using shear-wave ultrasound elastography. Am J Roentgenol. 2013;197:532-536. doi:10.2214/AJR.10.5449

16. Vergari C, Rouch P, Dubois G, et al. Non-invasive biomechanical characterization of intervertebral discs by shear wave ultrasound elastography: a feasibility study. Eur Radiol. 2014;24 (12):3210-3216. doi:10.1007/s00330-014-3382-8

17. Meng S, Reissig LF, Tzou CH, et al. Ultrasound of the hypoglossal nerve in the neck: visualization and initial clinical experience with patients. $A m$ J Neuroradiol. 2016;37:354-359. doi:10.3174/ajnr.A4494

18. Ye L, Qing Z, Homagi SR, et al. Ultrasound guided delineation of the recurrent laryngeal nerve and the tubercle of zuckerkandl. Methods Diagn Radiol J. 2019;1:1-5.

19. Roman BR, Randolph GW, Kamani D. Conventional thyroidectomy in the treatment of primary thyroid cancer. Endocrinol Metab Clin North Am. 2019;48:125-141. doi:10.1016/j.ecl.2018.11.003 


\section{Publish your work in this journal}

Cancer Management and Research is an international, peer-reviewed open access journal focusing on cancer research and the optimal use of preventative and integrated treatment interventions to achieve improved outcomes, enhanced survival and quality of life for the cancer patient.

The manuscript management system is completely online and includes a very quick and fair peer-review system, which is all easy to use. Visit http://www.dovepress.com/testimonials.php to read real quotes from published authors.

Submit your manuscript here: https://www.dovepress.com/cancer-management-and-research-journa/ 